\title{
Gestión del Conocimiento en la Industria de la Construcción: Estudio de un caso
}

\author{
Bahoque, Evila* \\ Gómez, Oda** \\ Pietrosemoli, Licia ${ }^{\star \star *}$
}

\section{Resumen}

El propósito de este artículo es explorar el proceso de gestión del conocimiento en la industria de la construcción, mediante los siguientes objetivos específicos: identificar el acceso del conocimiento a través del ámbito externo, interno e individual, conocer las herramientas utilizadas e identificar la plataforma sobre la cual se apoya y ejecuta la gestión del conocimiento en la empresa. La metodología utilizada es de tipo descriptiva, apoyada en la revisión documental de las teorías en el área, complementado con la aplicación de una encuesta y de entrevistas al personal de la organización. Los resultados obtenidos evidencian que en el caso estudiado de la industria de la construcción, la gestión del conocimiento forma parte del plan estratégico de la empresa, con miras a preservar y aprovechar los mismos; sin embargo, se carece de herramientas específicas y aplicaciones computarizadas especializadas para apoyar la elaboración de modelos de gestión del conocimiento. Con base en estos resultados, se recomienda formular estrategias y políticas de acción que permitan el desarrollo del modelo en beneficio del negocio, en términos de un mayor valor agregado, incremento de la competitividad y por ende, el fortalecimiento de la industria en el mercado nacional así como su incursión en mercados internacionales.

Palabras clave: Gestión del conocimiento, PyME, valor agregado, competitividad, industria de la construcción.

* Magister en Gerencia de Mantenimiento. Profesora Titular de la Facultad de Ingeniería de la Universidad del Zulia (LUZ). E-mail: ebahoque@cantv.net.

** Magister en Gerencia de Empresas Mención Finanzas. Profesora Titular de la Facultad de Ciencias Económicas y Sociales de la Universidad del Zulia (LUZ).

E-mail: odagomez@cantv.net.

*** Magister en Gerencia de Empresas Mención Finanzas. Profesora invitada de la Facultad de Agronomía de la Universidad del Zulia (LUZ). E-mail: licia_p@costanorte.com.ve 


\title{
Knowledge Management in the Construction Industry: a Case Study
}

\begin{abstract}
The purpose of this article is to explore the knowledge management process in the construction industry using the following specific objectives: identifying access to knowledge through external, internal and individual environments, knowing the tools used and identifying the platform on which the business's knowledge management is supported and carried out. The methodology used is of a descriptive type, supported by a documentary review of theories in the area, complemented by the application of a survey and interviews of the organization's personnel. Results show that in the case studied in the construction industry, knowledge management is a part of the company's strategic plan, with an eye to preserving and taking advantage of it; however, it lacks specific tools and specialized computer applications for supporting the development of knowledge management models. Based on these results, the study recommends formulating action strategies and policies for developing a model to benefit the business in terms of greater added value, increasing its competitiveness and therefore, strengthening the industry in the national market as well as its entry into international markets.
\end{abstract}

Key words: Knowledge management, SaME, added value, competitiveness, construction industry.

\section{Introducción}

Los cambios que se han producido en el entorno empresarial, caracterizado por la globalización de la economía, los avances en cuanto a tecnología de la información y las comunicaciones, así como los niveles de competitividad alcanzados en los diferentes sectores de la industria, han despertado el interés por la gestión del conocimiento, para establecer estrategias que permitan obtener ventajas competitivas en el desarrollo de sus operaciones y en sus resultados, convirtiéndose en una de las herramientas fundamentales de la gestión empresarial.

La gerencia del conocimiento ha sido aplicada en empresas grandes con poder económico, lo cual les permite la realización de proyectos para maximizar el valor agregado al conocimiento del re- curso humano, sin embargo en cuanto a las pequeñas y medianas empresas (PyMES), existen limitaciones económicas y estructurales para implantar un modelo de gestión del conocimiento, teniendo ellas que competir inclusive en el mismo mercado. Puesto que las PyMES no escapan del proceso de la globalización y se enfrentan a competidores internacionales, igualmente deben contar con estrategias sólidas, que permitan amortiguar la falta de recursos económicos, tecnológicos y humanos.

En Venezuela se estima que el $90 \%$ de la industria corresponde a PyMES, en donde la mayoría se encuentran en los sectores de comercio y servicios, y sólo un $14 \%$ son industrias manufactureras. Dada la importancia que tienen las PyMES, por su contribución al crecimiento de la economía nacional y 
que el conocimiento es una de las fuentes para que las organizaciones obtengan ventajas competitivas, se consideró pertinente seleccionar una empresa del sector de la construcción que presta servicio a la industria petrolera, con el objeto de explorar el proceso de gestión del conocimiento y conocer las actividades inherentes al mismo.

Partiendo del marco organizacional de la empresa objeto de estudio se identificó el acceso del conocimiento en el ámbito externo, interno e individual, así como las herramientas utilizadas para la aplicación de la gestión del conocimiento y la plataforma sobre la cual se apoya la misma.

Para la elaboración de este trabajo se utilizó una metodología de tipo descriptiva, apoyada en la revisión documental de las teorías en el área, complementado esto con la aplicación de una encuesta y de entrevistas al personal de la organización objeto de estudio, efectuadas en diciembre del dos mil cinco.

\section{Marco organizacional de Costa Norte Construcciones, C.A.}

Costa Norte Construcciones, C.A. (CNC), inició sus operaciones el 03 de Junio de 1.968 en el Estado Zulia, en la actualidad es una empresa de mediano tamaño y se desempeña en el campo de la construcción, mantenimiento y montaje. Sus bases de operaciones se encuentran en zonas cercanas a los centros de mayor interés para la Industria Petrolera, $\mathrm{Pe}$ troquímica, Carbonífera Nacional en los Estados Zulia, Falcón y Anzoátegui. Simultáneamente, la empresa ha realizado inversiones en un variado parque de maquinarias, equipos y herramientas industriales, hardware y software que le permiten atender los requerimientos de sus clientes.

La empresa ejecuta obras de construcción y mantenimiento industrial en las especialidades civil, mecánica, eléctrica, de instrumentación, izaje de equipos pesados y asesorías técnicas. Presta sus servicios principalmente a empresas nacionales e internacionales vinculadas a la industria petrolera, petroquímica y minera venezolana, y en menor escala, a otras entidades públicas y privadas.

En sus 38 años de actividad, la empresa ha desarrollado un know how en el campo de las construcciones petroleras y petroquímicas, que le han permitido ser reconocida como una empresa confiable para sus clientes. La empresa obtuvo en el año 2000 la certificación ISO 9000:1995 para sus actividades de Construcción y Montaje y en el año 2004 se recertificó bajo la norma ISO 9001:2000.

La misión de la empresa, ha sido definida así:

"Somos una empresa de construcciones que se propone brindar a los clientes el mejor servicio que puedan requerir, a través de la puesta en práctica de ideas y soluciones innovadoras a sus problemas. Para lograrlo, mejoramos constantemente nuestros niveles de ejecución y servicio.

Estamos comprometidos con el bienestar de las comunidades en las que estamos involucrados, conscientes de nuestra gran responsabilidad social. Este compromiso se materializa por la vía más permanente y digna que podemos ofrecer: fuentes de trabajo hones- 
tas para quienes quieran trabajar con mística y deseos de superación.

Nuestra filosofía empresarial y nuestra gestión gerencial se fundamentan en los siguientes valores: calidad total, honestidad, fidelidad y seriedad. Dentro de este marco buscamos recompensar a nuestros accionistas con un retorno justo y atractivo de su inversión."

La visión de la empresa, como:

"Ser la empresa de construcciones industriales más confiable para nuestros clientes, teniendo como base la calidad del servicio que préstamos, el cual estará al nivel de las empresas constructoras internacionales lideres del sector. Conseguir la máxima rentabilidad para nuestros clientes y accionistas gracias al aporte innovador a nuestras soluciones, a la utilización de nuevas tecnologías y a nuestro Sistema de Gestión de la Calidad COVENIN ISO9001:2000 en toda la empresa.

Ser reconocidos como una de las principales empresas del ramo, debido a la máxima productividad que alcanzamos en cada uno de los trabajos encomendados; por los beneficios tangibles que ofrecemos en cada uno de nuestros servicios y a la garantía de la calidad requerida por nuestros clientes.

Alcanzar el reconocimiento internacional como empresa líder en el izaje de equipos pesados y expandir nuestras operaciones en el Caribe y en América del sur".

Los objetivos de la empresa se han establecidos de acuerdo a la ejecución en el tiempo, en corto, mediano y largo, los cuales se describen a continuación.

Dentro de los objetivos a corto plazo la empresa se plantea: cumplir con los requerimientos de los clientes, asegurando la adecuada rentabilidad de cada proyecto y los niveles de seguridad idóneos, a saber: Incrementar la competitividad organizacional, incrementar los niveles de adiestramiento y formación del personal y mantener estándares de calidad correspondientes a la certificación bajo la norma ISO 9001-2000 obtenida.

\section{Los objetivos a mediano plazo} contemplan: mantener procesos de mejora continua, iniciar procesos de participación en los mercados internacionales, e incorporar a la organización en los procesos de cambio de las comunidades mediante mecanismos de proyección comunitaria orientada a la creación de una mentalidad favorable al esfuerzo individual y el trabajo creativo.

\section{A largo plazo, la empresa desea:} mantener el reconocimiento obtenido de ser una contratista integral, con visión del negocio y recursos humanos idóneos, desarrollar capacidades de ingeniería que permitan acumular conocimientos tecnológicos, ofrecer al mercado nuevos productos y procesos, consolidar una estrategia de mercadeo nacional e internacional activa, acorde a las características del mercado globalizado, y dominar el mercado venezolano mediante el desarrollo de una estrategia de servicios basados en calidad, competitividad e innovación.

Los aspectos que sostienen la aceptación de la oferta de servicios de CNC por parte del mercado son: la capacidad de respuesta de ejecución de obras, por disponer de infraestructura física y equipamiento para acometer obras de construcción y montaje; poseer valores y una cultura organizacional reconoci- 
dos por el mercado así como experiencia y disponibilidad de personal clave; además de gozar de credibilidad como entre sus clientes y relacionados. La empresa tiene presencia en las principales zonas de producción de hidrocarburos del país y amplia experiencia por la ejecución de múltiples proyectos para empresas petroleras y petroquímicas nacionales e internacionales.

\section{Diagnóstico de la Gestión del Conocimiento}

Davenport en 1998 definió el conocimiento como "la fluida mezcla estructurada de experiencia, valores, información contextualizada y ojo clínico muy experto que proporciona un marco de trabajo excelente para evaluar e incorporar nuevas experiencias e información", lo cual presupone que el conocimiento debería tener un carácter formal.

De hecho, el conocimiento informal siempre ha existido en las empresas, sin embargo la formalización de la gestión del conocimiento permite una mejor comunicación dentro de la organización y su entorno, conociendo oportunamente sus amenazas y oportunidades, así como sus fortalezas y debilidades, para tomar las acciones correctivas y mantener sus ventajas competitivas. De esta forma una gestión del conocimiento consciente y planificado, permite aprovechar adecuadamente el valor de los activos intangibles de la organización y su entorno.

Belly (2005), uno de los pioneros latinoamericanos en la gestión del conocimiento, indica que en la era industrial se gestionaban recursos tangibles, mientras que a partir del surgimiento de la era del conocimiento, en los años noventa, los principales recursos que se gestionan son intangibles. Señala igualmente, que el concepto de gestión como se entendía en la era industrial, no ha cambiado significativamente, a pesar del cambio tan radical en el tipo de recursos objetos de esa gestión. Menciona que inicialmente la gestión del conocimiento tenía como objetivo gestionar en una forma básica los conocimientos adquiridos por las personas de grupos heterogéneos; actualmente ese concepto ha sido ampliado hasta llegar al punto de que la gestión del conocimiento pretende agregar valor a la información disponible, mediante el almacenamiento, captación y difusión de ese mismo valor agregado en la organización. Esto ha permitido valorar el conocimiento como el principal activo de muchas organizaciones y fuente de importantes ventajas competitivas.

Para Takeuchi et al. (2004) la gestión del conocimiento es actualmente el verdadero centro de atención de la gerencia, puesto que es la que permite a las organizaciones adaptarse al entorno cambiante que las rodea y con ello sobrevivir en un ambiente altamente competitivo.

Garrido (2002), menciona que el ciclo del conocimiento mostrado en la Figura 1 , permite el proceso de externalización, que comprende la creación y clasificación de documentos dando origen al conocimiento explícito de tal forma, que éste puede ser extraído posteriormente por cualquier trabajador interesado, dando paso al proceso de internalización.

De acuerdo a los conceptos antes mencionados sobre la gestión del conocimiento se puede concluir que es una herramienta empresarial, que la administra- 
ción de las empresas debe conocer, organizar y evaluar, con el objeto de que este conocimiento sea aplicado adecuadamente en todos los procesos de la organización, de tal forma que permita generar valor con los activos intangibles de la organización. En tal sentido se consideró pertinente conocer la gestión del conocimiento en la empresa objeto de estudio, identificando el conocimiento desde tres ámbitos como son: externo, interno e individual, tal como se describe a continuación.

Dentro del ámbito externo se encuentran los elementos fundamentales de conocimiento que la organización necesita para crecer y mejorar, provenientes de: clientes, proveedores, competidores, gobierno, organismos reguladores, asociaciones empresariales, consultores, fuentes estadísticas, bancos y accionistas, mediante acuerdos de cooperación y alianzas estratégicas referidas a tecnologías, producción y comercialización.

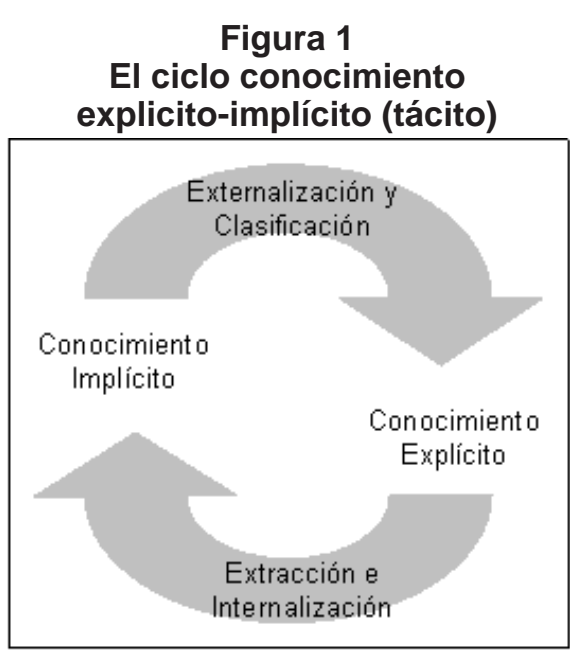

Fuente: Nonaka. The Knowledge Creating Company. Harvard Review.
En el ámbito interno se tiene la base del conocimiento de la empresa y sus experiencias, lo cual se puede ver reflejado en patentes, licencias, marcas, métodos/procedimientos de trabajo, software, bases de datos, sistemas de dirección, cultura de la empresa y en los procesos asociados a las áreas funcionales como son: control de calidad, producción, servicio, ingeniería, diseño, investigación y desarrollo, finanzas, entre otros.

Al ámbito individual corresponde la capacidad que posee el personal para realizar los procesos inherentes a la empresa, como son: educación, know how, conocimientos, habilidades, valores y actitudes de las personas.

Para efectos de este trabajo se decidió conocer estos ámbitos del conocimiento de acuerdo a los cuatro enfoques indicados por Rivero (2002), como son: cultural-social, metodológicos-operativos, organizativos y apoyo tecnológico, los cuales fueron considerados para clasificar las preguntas de la encuesta denominada "Diagnóstico de la Gestión del Conocimiento en CNC" aplicada al nivel directivo y ejecutivo. La puntuación máxima en cada enfoque resulta de ponderar el número de preguntas, el valor máximo de la escala (10) y la muestra considerada (8 gerentes). La puntuación obtenida se corresponde a la opinión de los gerentes encuestados. Ver Tabla 1.

En la Figura 2 se observa que los aspectos cultural-social, metodológicosoperativos y la plataforma tecnológica poseen un nivel de desarrollo similar $(61.8 \%$ y $63.75 \%$, respectivamente), consistente con el esfuerzo aplicado por la empresa para valorar el conocimiento, lo cual representa un nivel satisfactorio, de acuer- 


\section{Tabla 1 \\ Resultados de la encuesta sobre el Diagnóstico de la Gestión del Conocimiento en CNC}

\begin{tabular}{lcccc}
\hline & $\begin{array}{c}\text { No. de } \\
\text { Preguntas }\end{array}$ & $\begin{array}{c}\text { Puntuación } \\
\text { Máxima }\end{array}$ & $\begin{array}{c}\text { Puntuación } \\
\text { Obtenida }\end{array}$ & $\begin{array}{c}\text { Puntuación } \\
\text { Obtenida (\%) }\end{array}$ \\
\cline { 2 - 5 } 1. Culturales y sociales & 57 & 4.560 & 2.818 & $\mathbf{6 1 . 8 0}$ \\
2. Metodológicos y operativos & 27 & 2.160 & 1.222 & $\mathbf{5 6 . 5 7}$ \\
3. Enfoque Organizativo & 7 & 560 & 220 & $\mathbf{3 9 . 2 9}$ \\
4. Apoyo tecnológico & 7 & 560 & 357 & $\mathbf{6 3 . 7 5}$ \\
\hline
\end{tabular}

Fuente: Elaboración propia con base en los resultados obtenidos en la encuesta.

\section{Figura 2 \\ Diagnóstico de la Gestión del Conocimiento}

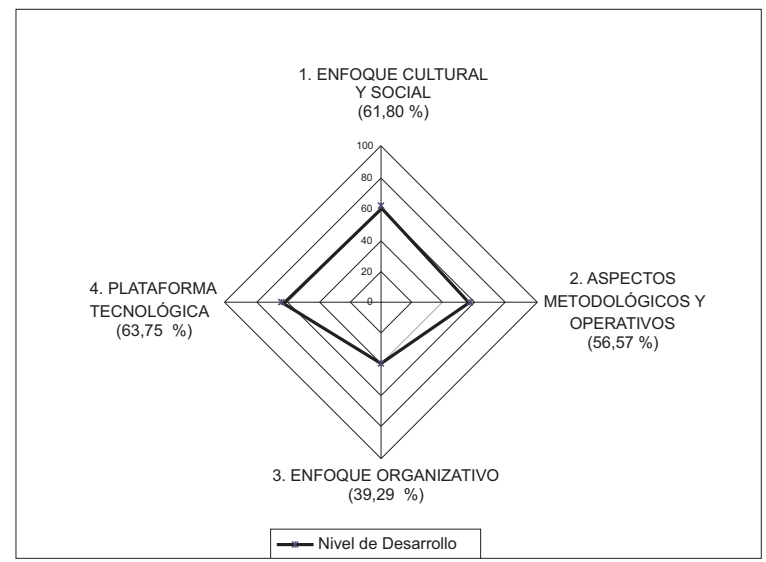

Fuente: Elaboración propia basado en el Cuadro 1.

do a los planes. El aspecto que requiere mayor atención es el organizativo, en el sentido de formalizar la gestión del conocimiento mediante el desarrollo de un modelo. A continuación se presenta un análisis de cada uno de estos enfoques, en el caso objeto de estudio.

\subsection{Aspecto cultural y social}

El objetivo es medir la presencia de elementos que favorecen la existencia de un adecuado sistema de gestión del conocimiento. El caso estudiado refleja un $61.8 \%$ en la cultura organizacional, lo cual implica la presencia implícita de la gestión del conocimiento, concebida en forma tácita dentro de los principios organizacionales.

Los resultados de la encuesta así como la misión, la visión y los valores organizacionales, evidencian el objetivo de la empresa de crear un nuevo ambiente de trabajo, caracterizado por un esquema 
de cooperación del conocimiento. De este modo, la empresa concientemente propicia que las personas apliquen sus conocimientos y sus experiencias como un equipo sinérgico capaz de innovar y de transformar los procesos ante los cambios del entorno. A nivel de la directiva y de la gerencia de la empresa, se reconoce la importancia del buen uso del conocimiento para lograr el éxito en las organizaciones, considerando que la información por sí misma no es suficiente, ya que sólo el conocimiento permite la diferenciación competitiva.

En CNC la cultura organizacional sirve de marco de referencia y da las pautas acerca de cómo sus directivos y empleados deben conducirse en ésta. Por otra parte, se evidenció que el actual sistema de gestión de la calidad ha permitido organizar, recopilar y poner a disposición de todos los miembros de la organización un cúmulo de conocimientos anteriormente dispersos y no formalizados. Este sistema en conjunto con la plataforma tecnológica, los planes de adiestramiento y los sistemas de comunicación, ha conducido a la empresa a disponer de conocimientos tácitos o implícitos, que comprenden las experiencias personales de aprendizaje acumuladas en los 38 años de actividades y un conocimiento explícito que se estructura, almacena, comunica y distribuye dentro de la organización. Ambos son reconocidos como recursos estratégicos, que deben ser usados en forma efectiva, mediante canales para la captación del conocimiento.

En base a ello se concluye que la empresa objeto de estudio tiene un basamento cultural y social que propicia la generación, administración, difusión, preservación y asimilación del conocimiento en todos sus niveles, existiendo consenso en cuanto a la necesidad de documentar y compartir el conocimiento individual y que este sea reconocido como una de las fortalezas de la organización. Esta práctica ha creado un proceso de retroalimentación en el que los resultados estimulan el uso y mejoramiento de los conocimientos y despiertan el interés para propiciar este proceso en todas las actividades.

\subsection{Aspectos metodológicos y operativos}

Para tener una visión global de los aspectos metodológicos y operativos de la gestión del conocimiento, estos fueron clasificados en tres fases, como son: existencia de un conocimiento estructural, asimilación del conocimiento por parte de los usuarios y utilización eficiente del conocimiento, lo cual determina las herramientas de gestión requeridas, permite conocer el inventario de los conocimientos disponibles y los requeridos, así como definir la forma de atender dichos requerimientos.

\section{Fase I: Existencia de un conoci- miento estructural}

Esta fase se refiere a las estrategias que la empresa aplica para proteger el conocimiento. La información obtenida evidencia la existencia de variados procesos formales orientados a gestionar el mismo, para alcanzar y mantener sus ventajas competitivas, por medio de la documentación de los procesos del sistema de gestión de la calidad para las líneas de construcción y montaje, certificado por FONDONORMA. Esta filosofía de 
gestión ha permitido a la empresa lograr la documentación progresiva de los conocimientos, así como su utilización sistemática como recurso estratégico. Los procesos de auditorias que realiza FONDONORMA como ente certificador, han apoyado la revisión, atención y asimilación de los conocimientos, puesto que los mismos además de estar disponibles, son utilizados para la mejora continua.

Los manuales de normas y procedimientos, especificaciones de productos o servicios, instrucciones de trabajo, manual de descripciones de cargos, mapas de procesos y otros elementos que forman parte del sistema de gestión de la calidad, son evidencias del conocimiento estructural que utiliza la empresa en sus actividades cotidianas. La Figura 3 , muestra la pirámide documental del sistema de la calidad y la Figura 4 los mapas de procesos generales de la organización.
Fase II: Asimilación del conocimiento por los usuarios

El proceso de asimilación del conocimiento debe realizarse atendiendo a los aspectos básicos de dicho proceso y a la gestión del mantenimiento de dicho conocimiento, para lo cual se requiere la detección de necesidades, el desarrollo de un plan para la transmisión y asimilación del conocimiento, así como la asignación de los recursos necesarios para dichos planes.

En esta fase se observó que la empresa objeto de estudio adquiere conocimientos a través de fuentes muy diversas, entre las cuales resaltan los servicios de prensa local y nacional, el resumen de prensa de la Cámara Petrolera del Zulia e información de otros organismos como la Cámara de Comercio, Cámara de Industriales, Cámara de la Construcción, Asociación Venezolana de Exportadores de Construcción (AVEX-

\section{Figura 3 \\ Pirámide documental del Sistema de Calidad}

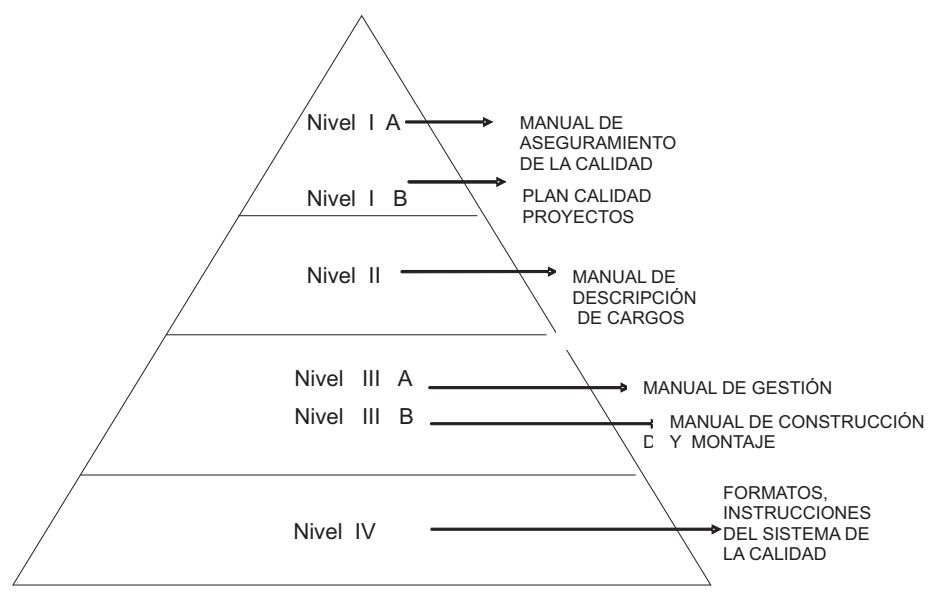

Fuente: Manual de Calidad de Costa Norte Construcciones. 


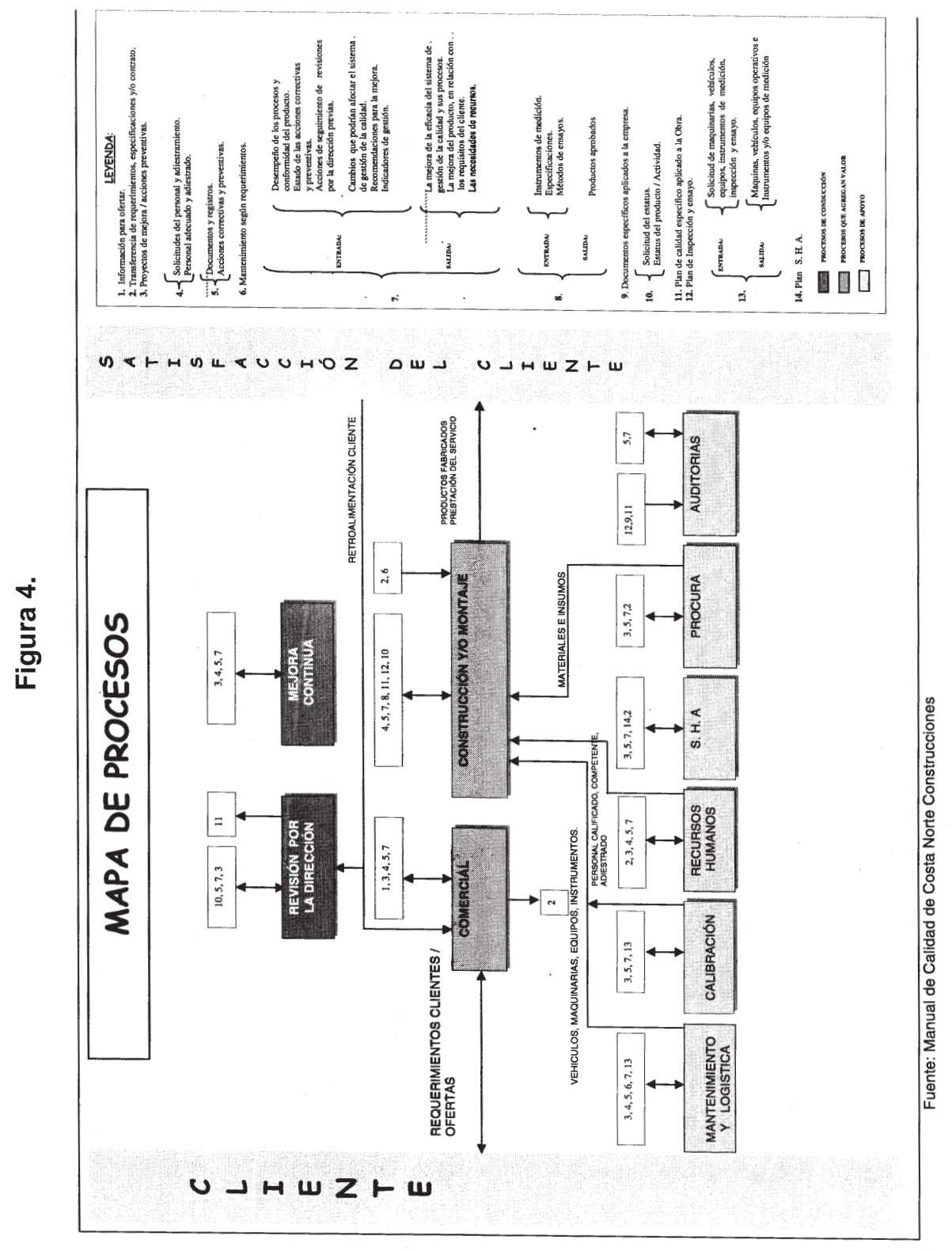


CON), Comisión de Administración de Divisas (Cadivi), SENIAT, boletines de empresas consultoras y la banca nacional.

Los directivos y gerentes de la empresa asisten a reuniones con clientes, así como a foros, congresos y exposiciones relacionadas con las distintas gerencias. En estos eventos se obtiene información sobre las expectativas de los clientes, de los organismos relacionados y de la competencia. Ocasionalmente, la empresa contrata estudios de mercado especializados para atender alguna necesidad específica y no utiliza patentes propias ni de terceros.

Adicionalmente la empresa cuenta con equipos de asesores en las áreas: técnica, comercial, contable, financiera, fiscal, laboral, legal e informática, quienes la mantienen actualizada sobre los cambios sufridos en las distintas áreas de acción y/o nuevas tecnologías, logrando que la organización pueda mantener su capacidad de respuesta a los nuevos requerimientos y tendencias del mercado, todo lo cual indica que la empresa hace esfuerzos importantes para absorber e incorporar a sus procesos los conocimientos que se generan en el entorno.

Fase III: Utilización eficiente del conocimiento

La gestión del conocimiento debe tener como meta el uso eficiente del conocimiento para el mejoramiento continuo y adecuación de sus procesos. A través de las encuestas, revisión de documentación y entrevistas, se observó que una parte importante de los procesos críticos de la empresa, tales como recursos humanos, tesorería, cuentas por cobrar, cuentas por pagar y contabilidad se manejan en tiempo real, lo cual permite tener información inmediata sobre la situación y resultados, así como disponer en forma oportuna de indicadores de gestión para la retroalimentación de los procesos.

En cuanto a los procesos de construcción, es importante resaltar el valor agregado del manejo de la información en tiempo real, ya que es posible tener respuestas oportunas que permitan medir resultados y detectar desviaciones respecto a lo planificado.

A pesar de los avances en la materia, la organización tiene otros procesos de valor agregado, tales como licitaciones, planificación, control de proyectos, compras y almacén, mantenimiento y logística, que no están en línea sino que se controlan basados en información histórica. En cuanto a los manuales, sólo el manual de contabilidad y los manuales del sistema de la calidad, están en línea.

Para proteger la información relacionada con el sistema de la calidad y el sistema financiero, los niveles directivo y gerencial han establecido controles, mediante la firma de convenios de confidencialidad con asesores externos y subcontratistas para casos específicos, estableciéndose restricciones de uso de la información, así como lineamientos para el acceso y extracción de información. Para el resto de los casos, la empresa no tiene normas específicas de preservación del conocimiento.

En materia de apoyo tecnológico, la empresa tiene establecidos mecanismos formales de respaldo automático y periódico de la data e información contenida en los sistemas y aplicaciones. La información es respaldada a nivel nacional en los diversos servidores, por lo cual se garantiza que cada división operativa, 
siempre tenga un respaldo en otra división. Adicionalmente la empresa tiene establecido un plan de seguridad de barreras (firewall) y claves de acceso, que permiten proteger los conocimientos almacenados en los distintos recursos tecnológicos. Además, la gerencia de sistemas monitorea las distintas aplicaciones a nivel nacional para detectar uso inadecuado de los recursos o accesos no autorizados a algunas aplicaciones.

\subsection{Enfoque organizativo}

El enfoque organizativo propone emular en un modelo, la importancia de una visión integral de los procesos de gestión del conocimiento. Por ello sugiere la evaluación de los mapas y procesos que la empresa pueda tener diseñados para este fin.

Dada la importancia que tiene hoy en día la gestión del conocimiento, este proceso debe ser análogo a otras actividades gerenciales y de administración, cuyos objetivos permitan: identificar, detectar, reelaborar y poner a disposición los conocimientos y las prácticas que aporten valor agregado. La responsabilidad por la aplicación de las políticas y normas es de los administradores de línea, mientras que los procesos de análisis, apoyo, identificación de oportunidades, entre otros, deberán compartirse con una unidad de alcance corporativo especializada en el tema, tal como ocurre en la administración del resto de los recursos empresariales.

En esta materia, tal como se deduce del análisis de los resultados de las encuestas y revisión de la documentación disponible, la empresa estudiada carece de herramientas específicas y de educación formal para el modelado de los procesos de gestión del conocimiento. Esto se evidencia en el hecho de que a pesar de que la organización maneja los conocimientos como uno de sus principales recursos estratégicos, aún carece de un sistema formal que permita a los agentes, simular eventos que pueden suceder a partir de los conocimientos actualmente disponibles. En resumen, la empresa carece de herramientas específicas y aplicaciones computarizadas especializadas para apoyar la elaboración de modelos.

\subsection{Plataforma tecnológica}

La gestión del conocimiento no es sólo tecnología, pero en la era actual de la información y las comunicaciones, debe considerarse el rol que la plataforma tecnológica cumple en el logro de las metas en esta materia. En este sentido, Koulopoulos y Frappaolo (2000), afirman que el gerente inteligente debe determinar las tecnologías en las cuales invertir, y cómo esas tecnologías pueden ayudar a resolver las necesidades de conocimientos de la organización. Una auditoria sobre el conocimiento y un mapa de necesidades de la organización, darán orientación para resolver estos aspectos. Estos autores lo definen como el knoware (software del conocimiento) y sus componentes están asociados a: bases de datos, bases de conocimientos, manejo de documentos, intranet, extranet, software de grupo (Groupware), software de colaboración, herramientas de búsqueda, herramientas de navegación, apoyo de decisiones e inteligencia artificial, flujo de trabajo, Internet, Web y multimedia. En el Cuadro 1 , 
muestra como algunas tecnologías pueden contribuir en la transición de conocimientos tácitos a conocimientos explícitos y viceversa, en los diferentes niveles de gestión del conocimiento.

Según Garrido (2000), para facilitar el flujo de conocimiento para el proceso de creación, tanto de conocimiento explícito como implícito, se han desarrollado una serie de herramientas tecnológicas, las cuales deben entenderse dentro del entorno tecnológico donde operan, estos avances deben acoplarse a los sistemas ya existentes.

El entorno tecnológico, además de sistemas de software, también incluye sistemas de hardware, desde mainframes hasta computadores personales, servidores y dispositivos portátiles. Tanto las tecnologías del software como las de hardware deben estar diseñadas con el propósito de disminuir la distancia comunicativa y proveer un entorno común para el almacenaje, acceso y compartimiento del conocimiento.
Para poder reutilizar el conocimiento es necesario disponer de herramientas de clasificación, búsqueda, almacenamiento y extracción. Con este propósito se han diseñado múltiples herramientas de software que facilitan estos procesos, las cuales van más allá de las simples herramientas de datos o gestión de información, debido a que comprenden tareas más complejas, permitiendo la interacción efectiva entre los miembros de la organización.

En concordancia con lo anterior, la empresa objeto de estudio posee una plataforma tecnológica que sustenta los procesos de gestión del conocimiento, como se observa en la Figura 5. La gerencia de sistemas de información es la encargada de apoyar la generación, comunicación, intercambio, difusión, utilización y preservación del conocimiento de los ámbitos interno y externo. Dicha plataforma funciona a través de una intranet o red de área amplia para uso interno, que enlaza a la organización a nivel na-

\section{Cuadro 1}

\section{Tecnologías según nivel de gestión y complejidad del conocimiento}

\begin{tabular}{|c|c|c|c|}
\hline \multirow{5}{*}{ 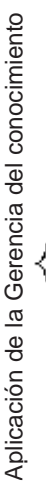 } & Explícito & \multicolumn{2}{|l|}{ Complejidad del o } \\
\hline & Intermediación & $\begin{array}{l}\text { Groupware, manejo de documentos, } \\
\text { Correo electrónico }\end{array}$ & $\begin{array}{l}\text { Comunidades } \\
\text { de práctica }\end{array}$ \\
\hline & Exteriorización & $\begin{array}{l}\text { Manejo de documentos, búsqueda y } \\
\text { Recuperación, visualización, portales }\end{array}$ & Tutoría \\
\hline & Interiorización & $\begin{array}{l}\text { Búsqueda y recuperación, } \\
\text { Agentes }\end{array}$ & $\begin{array}{l}\text { Capacitación } \\
\text { de aprendices }\end{array}$ \\
\hline & Cognición & $\begin{array}{l}\text { Flujo de trabajo, apoyo de } \\
\text { Decisiones, visualización }\end{array}$ & Intuición \\
\hline
\end{tabular}

Fuente: Koulopoulos T.M., Frappaolo C. (2000). 


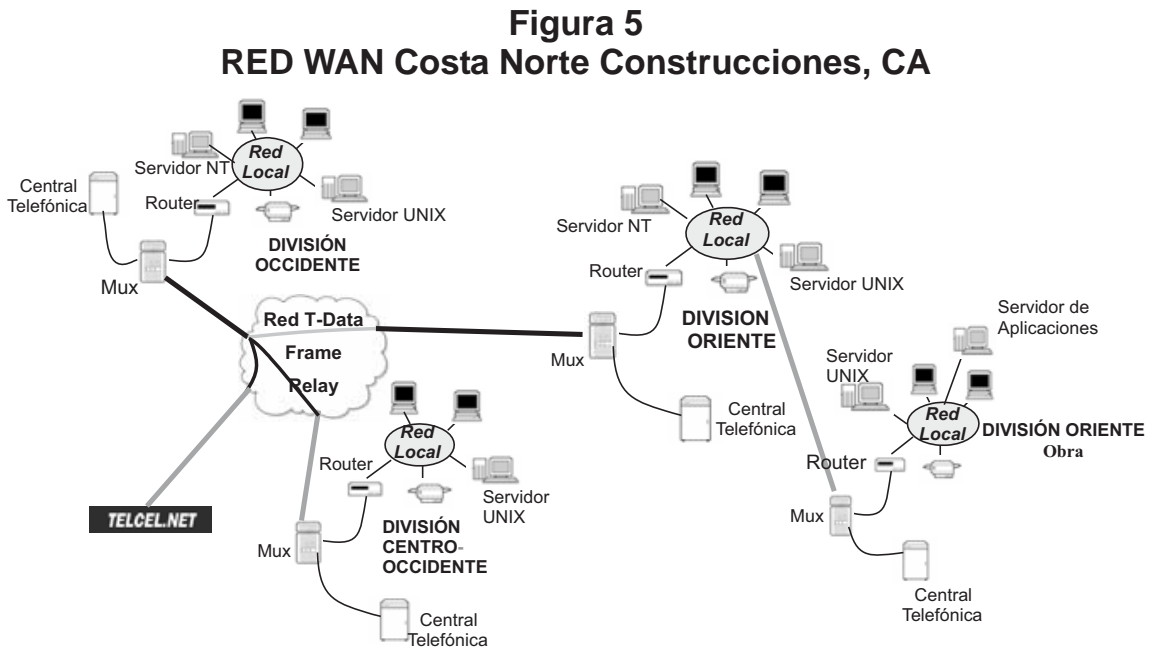

Fuente: Plan de negocios Costa Norte Construcciones C.A. (2004-2007).

cional, llegando incluso a nivel de los proyectos que se ejecutan en zonas diferentes de las bases operativas permanentes ubicadas en los Estados Zulia, Falcón y Anzoátegui.

Los sistemas de la empresa y la intranet utilizan el frame relay como canal de comunicaciones vía microonda, suplido por un proveedor externo, que brinda el servicio de transmisión de vozy datos a alta velocidad. Esta solución le resulta a la empresa confiable, de alto rendimiento y con una relación costo/beneficio adecuada. Sobre este servicio se integran y trabajan las soluciones de software comerciales y propias que emplea la empresa. El sistema operativo donde están instalados los sistemas de información de la empresa es Linux, que además de ser público resulta confiable y seguro.

Las soluciones propias que la empresa utiliza para recursos humanos, tesorería, cuentas por cobrar, cuentas por pagar, impuestos, mantenimiento logística, almacén y contabilidad están diseñadas bajo Foxpro para Linux, además utiliza otras aplicaciones comerciales tales como Microsoft Office, Primavera y otros programas, todos con licencias actualizadas.

Las soluciones desarrolladas para la empresa, han incorporado módulos de generación de indicadores de gestión que apoyan la toma de decisiones, los cuales están relacionados con las metas y objetivos establecidos en el sistema de la calidad, estos indicadores son revisados periódicamente por los gerentes y en las reuniones de Junta Directiva. Actualmente la empresa trabaja en el desarrollo de nuevas versiones a algunas soluciones, tales como almacén, mantenimiento y logística, para adecuarlas a los nuevos requerimientos de la organización.

De acuerdo a los resultados de las encuestas y la información disponible, la 
organización dispone de herramientas que apoyan la gestión del conocimiento mediante:

- Programas de gestión de la documentación y de imagen que permiten: la creación de bases de datos con referencias de documentos y sus contenidos; la digitalización de la información; la creación de bases de datos de conocimiento en diferentes áreas, facilitando la búsqueda por diversas vías y un tratamiento versátil de la información; y el enlace de la información contenida en un registro, con los documentos asociados situados en otros programas informáticos de la red o Intranet.

- Sistemas de edición electrónica, que permiten editar información de manera rápida y a bajo costo, haciéndola accesible a los trabajadores de la organización mediante la Intranet y más allá, a la sociedad en general mediante Internet. A futuro, se tiene previsto dentro del plan de mejoramiento, llegar a clientes, colaboradores, proveedores y relacionados en general, mediante una Extranet.

- Motores de búsqueda que permiten: la búsqueda de información, mediante exploración en direcciones de Internet en la propia Web y también en los programas informáticos de la misma empresa así como la creación de bases de datos.

- Sistemas interactivos: entre otras mejoras, la empresa inicia sus procesos para la compilación de documentos tales como encuestas y formularios, permitiendo el acceso a través de la Web, facilitando a los usuarios la completación de estos documentos, y su envío en forma directa e inmediata al servidor, integrando las respuestas en bases de datos definidas para su tratamiento estadístico.

Por ende se puede concluir que la plataforma tecnológica de la empresa estudiada, en términos de hardware y software, brinda facilidades para una adecuada gestión del conocimiento.

\section{Conclusiones}

De acuerdo a los resultados de la investigación realizada, hay evidencias de que en la empresa estudiada se han iniciado procesos formales de gestión del conocimiento, con miras a preservar y aprovechar los mismos. Se detecta la inversión de recursos en la difusión de los principios y valores corporativos que reconocen al conocimiento como un valioso activo organizacional, en el adiestramiento y formación del personal, en la recopilación, análisis y difusión del conocimiento del entorno y en la creación de una plataforma tecnológica que permita que el conocimiento sea empleado para el mejoramiento continuo del negocio.

En cuanto al enfoque cultural y social, fue calificado por parte del nivel directivo con un $61.80 \%$ de desarrollo, lo cual significa que algunos niveles de la organización reconocen objetivamente el valor estratégico del conocimiento, entre ellos la Gerencia de Aseguramiento de la Calidad, la Gerencia de Recursos Humanos, Contraloría Interna y Proyectos. En otros niveles de la organización, el reconocimiento y valoración del conocimiento, debe ser formalizado e integrado, tanto vertical como horizontalmente. 
Los aspectos metodológicos y operativos fueron calificados en un $57.56 \%$, debido principalmente a que la empresa está certificada con la ISO 9001-2000, no obstante la implantación de la gestión del conocimiento está aún en proceso y está contenida en el plan de negocios 20042007.

En lo organizativo existe un desarrollo del $39.29 \%$, debido principalmente a que la empresa, tiene definidos los mapas de los procesos medulares, pero no posee un modelo formal de gestión del conocimiento, ni herramientas específicas y aplicaciones computarizadas especializadas para apoyar la elaboración de dicho modelo.

La empresa cuenta con una plataforma tecnológica en evolución, que permite el control de los procesos y el desarrollo de una cultura organizacional, en la cual la información es valorada tanto como otros recursos utilizados y es empleada para la planificación, control de actividades y retroalimentación de los procesos, de manera que los mismos mantengan el ciclo de mejora continua para mejorar los niveles de competitividad, lo cual sitúa este aspecto con una valoración del $63.75 \%$ con posibilidades en el corto plazo de continuar esta tendencia favorable.

En general, Costa Norte Construcciones C.A. está consciente de la importancia de la gestión del conocimiento y ha logrado avances importantes en este sentido, todos previstos en su plan estratégico de negocios. Con el objeto de apoyar los avances en esta área, se recomienda a la empresa formular estrategias y políticas de acción que permitan el de- sarrollo de un modelo formal de gestión del conocimiento en beneficio del negocio, en términos de un mayor valor agregado, incremento de la competitividad y por ende, el fortalecimiento de la industria en el mercado nacional así como su incursión en mercados internacionales.

\section{Referencias Bibliográficas}

Albornoz, Orlando (2000). La gerencia del conocimiento y la competitividad académica en la educación superior. Consultado el 21 de Noviembre de 2005. Disponible en: http://www. aprender.org.ar/aprender/articulos

Belly, Pablo (2005). Gestión del Conocimiento y Capital Humano Consultado 20 de Noviembre del 2005, en http://.gestiopolis.com/canales/gerencial/artículos/59/origen.htm

Costa Norte Construcciones C.A. (2003). Manual de aseguramiento de la calidad. Maracaibo - Venezuela.

Costa Norte Construcciones C.A. (2005). Manual de gestión de procesos. Maracaibo - Venezuela.

Costa Norte Construcciones C.A. (2004). Plan de Negocios 2004-2007. Maracaibo - Venezuela

Davenport, T.H., Prusak, L. (1998). Working Knowledge: How Organizations Manage What They Know", Boston: Harvard Business School Press.

Garrido Castillo, Ruth del Valle (2002). Diseño de un modelo de gestión del conocimiento para la Unellez que promueva el desarrollo de ventajas competitivas en el área de investigación. Tesis Doctoral UNELLEZ, Barinas, Estado Barinas, Venezuela.

Guèdez, Víctor (2003). Aprender a emprender. De la gerencia del conocimiento a la ética de la sabiduría. Editorial Planeta Venezolana. S.A. 
Koulopoulos T.M.; Frappaolo, C. (2000). Lo fundamental y más efectivo de la Gerencia del conocimiento. Editorial Mc Graw-Hill.

Quintas, Paul; Lefrere, Paul; Jones, Geoff (1997). "Knowledge Management: a Strategic Agenda", Long Range Planning, Vol. 30, No. 3, Elsevier Science Ltd.
Rivero Rodrigo, Santiago (2002). La gestión del conocimiento. Editorial Socintec. España.

Takeuchi Hirotaka, Nonaka, Ikujiro (2004). Hitotsubashi on Knowledge Management. Jhon Wiley \& Sons Asia. Singapore. 